\title{
Software studies, o processo criativo \\ e as poéticas do digital ${ }^{1}$
}

\section{Daniel Peixoto Ferreira}

Universidade de São Paulo

danielpferreira@yahoo.com.br

\section{Prof. Dr. Gilbertto dos Santos Prado}

Universidade de São Paulo gttoprado@ajato.com.br

\section{Estudos do software}

A disciplina dos Estudos do Software (Software Studies) é uma área de pesquisa relativamente nova e de caráter unificador, reunindo sob um mesmo campo de conhecimento determinados conceitos, autores e linhas de pesquisa que compartilham o enfoque cultural no estudo do software, da sua lógica e dos sistemas a ele relacionados.

Enquanto a Ciência da Computação e a Engenharia de Software enfatizam os aspectos técnicos e práticos dos algoritmos e da tecnologia, os Estudos do Software promovem uma discussão a respeito dos significados que emergem do relacionamento entre o humano e o

\footnotetext{
${ }^{1}$ Título em inglês: "Software Studies, the Creative Process and the Poetics of Digital" (a tradução "poetics of digital" busca refletir o sentido pretendido pelo termo em português "poéticas do digital", que difere de "poéticas digitais" (em inglês, "digital poetics"), como explicamos mais adiante neste artigo)
} 
numérico em outros contextos, mais amplos e variados - desde o social, passando pelo econômico e histórico, até o da criação artística.

Evidentemente existem autores que adotam uma postura similar a esta mas que não se encontram diretamente associados à iniciativa dos Estudos do Software, bem como autores cuja atuação precede a sua própria formação. De qualquer forma, a fundamentação teórica desta disciplina é útil como referência para esta abordagem específica ao estudo do software e da tecnologia de uma maneira mais ampla.

\section{Origem}

O termo "estudos do software" foi empregado pela primeira vez por Lev Manovich em The Language of New Media (2001). No trecho a seguir ele apresenta o que futuramente viria a se tornar esta nova disciplina:

Para entender a lógica das novas mídias precisamos nos voltar à Ciência da Computação. É aí que podemos esperar encontrar os novos termos, categorias e operações que caracterizam as mídias que se tornaram programáveis. Dos estudos de novas mídias, passamos a algo que pode ser chamado de estudos do software; da teoria das mídias - à teoria do software.2 (MANOVICH, 2001:65)

O autor deixa clara a característica principal que define as novas mídias: a programabilidade do meio numérico. Assim, se por um lado a capacidade de armazenagem e velocidade de acesso aos dados e mesmo a interatividade são elementos significativos nas mídias digitais, residiria

\footnotetext{
2 Ênfase do autor (sublinhado no texto original). Texto original: "To understand the logic of new media we need to turn to computer science. It is there that we may expect to find the new terms, categories and operations which characterize media which became programmable. From media studies, we move to something which can be called software studies; from media theory - to software theory." (todas as traduções neste artigo são nossas, a partir do inglês)
} 
no aspecto procedimental o diferencial das novas mídias sobre as mídias tradicionais3.

Esta mudança é tão significativa que Manovich sugere a necessidade de uma área de estudos específica, que forneça os mecanismos teóricos e operacionais necessários para lidar com esta nova situação que se apresenta.

\section{Principais autores}

Lev Manovich é autor e palestrante na área de novas mídias, professor de artes visuais na Universidade da Califórnia (San Diego UCSD) e diretor da iniciativa Software Studies na Calit2 (California Institute for Telecommunications and Information Technology).

Assim como muitos artistas e autores na área de novas mídias, Manovich teve o seu percurso artístico e acadêmico marcado pela interdisciplinaridade. $\mathrm{O}$ autor estudou belas artes antes de se interessar pelas possibilidades criativas da tecnologia, primeiro através da computação gráfica, depois na programação de computadores.

Outros pesquisadores atuando diretamente na área são Noah Wardrip-Fruin (diretor associado ao lado de Manovich na Calit2) e, coordenando a iniciativa dos Estudos do Software no Brasil, Cicero Silva, professor no Instituto de Artes e Design da Universidade Federal de Juiz de Fora.

\footnotetext{
${ }^{3}$ Segundo Michael Mateas, é o aspecto procedimental do meio numérico - isto é, a sua capacidade de conter e executar instruções e procedimentos determinados pelo usuário que o distingue das demais mídias e ferramentas tradicionais (MATEAS, 2005). De acordo com Janet Murray, além da procedimentalidade existem outros três aspectos que caracterizam o meio numérico: o participativo, o espacial e o enciclopédico (MURRAY, 2003) - para Mateas, porém, o aspecto principal é o procedimental, do qual todos os demais derivariam.
} 
Há diversos autores que, embora não se relacionem diretamente à disciplina dos Estudos do Software, compartilham de alguma forma da sua abordagem, entre eles John Maeda (Diretor Associado de Pesquisa do MIT Media Lab), Ben Fry e Casey Reas (criadores do Processing4) e Michael Mateas (diretor do Expressive Intelligence Studio na Universidade da California, em Santa Cruz).

\section{Série MIT Press}

Em 2008, a MIT Press deu início a uma série dedicada especificamente à disciplina dos Estudos do Software. O trecho a seguir faz parte do texto de apresentação da série, e sintetiza a linha editorial pretendida:

O software está profundamente entrelaçado com a vida contemporânea - economicamente, culturalmente, criativamente, politicamente - de maneiras tanto óbvias quanto praticamente invisíveis. Todavia, enquanto muito se escreve sobre como o software é utilizado, e as atividades que ele suporta e molda, o pensar sobre o software em si tem permanecido predominantemente técnico pela maior parte da sua história. 5 (FULLER et al., 2009)

O livro Software Studies: A Lexicon (FULLER, 2008) de certa forma inaugura esta série, contendo textos de diversos autores em diferentes áreas de pesquisa. Além de apresentar e explorar os fundamentos da disciplina, como sugere o título, a publicação serve como uma amostra das diferentes perspectivas e abordagens através das quais é possível estudar o software e a tecnologia de uma maneira mais ampla. Desde a

\footnotetext{
${ }^{4}$ A linguagem de programação Processing propõe uma abordagem numérica ao processo criativo, permitindo que mesmo artistas não familiarizados com algoritmos possam programar suas idéias em um ambiente de autoria dinâmico, intuitivo e extremamente flexível. Site: processing.org

5 Texto original: "Software is deeply woven into contemporary life-economically, culturally, creatively, politically-in manners both obvious and nearly invisible. Yet while much is written about how software is used, and the activities that it supports and shapes, thinking about software itself has remained largely technical for much of its history."
} 
análise do algoritmo como arte, passando pelos aspectos sociais e políticos relacionados ao software, até discussões sobre técnicas de compressão, formatos de arquivo e tecnologias de transmissão de dados sempre a partir de um enfoque reflexivo e cultural.

Matthew Fuller, editor do livro, explica que a intenção desta compilação é de causar um estranhamento perante a tecnologia, e principalmente de levar a uma postura reflexiva diante do software. Desta maneira, indo além das questões técnicas geralmente enfatizadas nas discussões nesta área, é possível adotar um ponto de vista menos alienado, mais crítico e informado.

O autor identifica dois extremos que considera perigosos no emprego dos meios digitais: de um lado, a pretensão de acreditar que através do software é possível dominar a realidade; do outro, a limitação de se render à terminologia de especialistas e realizar apenas o que já se conhece.

No texto de introdução do livro, Fuller resume com precisão um dos aspectos mais fortes do meio numérico, que é o seu potencial de servir como reflexo da própria mente humana, permitindo formalizar, experimentar e mesmo dialogar com modelos dos nossos próprios pensamentos: "Para programar, você precisa entender alguma coisa tão bem a ponto de ser capaz de explicá-la a algo tão tapado quanto um computador.6 (FULLER, 2008:10)"

\section{Software Takes Command}

Ainda em 2008, Manovich dá seguimento a The Language of New Media com Software Takes Command (2008). Lançado em um formato

\footnotetext{
6 Texto original: "In order to program, you have to understand something so well that you can explain it to something as stonily stupid as a computer." (utilizamos o termo "tapado" em nossa tradução na tentativa de emular a natureza informal da expressão utilizada no texto original - "stonily stupid")
} 
que o autor denomina como "softbook", ou "livro aberto" (em referência ao conceito de "software aberto" ou "software livre"), o texto foi disponibilizado online na íntegra para download. Além disso, Manovich encorajou a participação dos seus leitores, convidando-os a contribuir com material adicional, modificações e correções.

No texto, Manovich continua examinando as novas mídias que, embora não sejam exatamente novas, certamente ganham uma nova perspectiva através do olhar investigativo do autor. Também são examinados os aspectos sociais relacionados a estas práticas, tema cada vez mais onipresente e relevante, à medida que aumenta a importância e o papel da tecnologia no mundo contemporâneo.

Além disso, o autor investiga as motivações que guiaram os inventores e pensadores das novas mídias, passo fundamental para a compreensão tanto das origens quanto do futuro destas tecnologias7. 0 livro também trata das mudanças de paradigma causadas pelo processo de "softwarização" ("softwarization") da cultura, além da influência das novas interfaces e ferramentas - do que Manovich denomina como "Software Cultural" - na estética contemporânea8.

De acordo com o autor, o Software Cultural trata-se de uma categoria específica de aplicativos, pelos quais é possível criar e acessar objetos e ambientes midiáticos. Fazem parte desta categoria desde os populares Word, PowerPoint e Explorer (Microsoft) até aplicativos especializados, como a suíte da Adobe, Photoshop, Illustrator e Flash, entre outros.

\footnotetext{
${ }^{7}$ O resgate da história das novas mídias (e das tecnologias em geral) é uma preocupação constante de Manovich. Por conta da velocidade pela qual novas tecnologias, mídias e linguagens surgem e se transformam, a ausência de uma conexão com o passado se torna especialmente evidente nesta área.

8 É possível traçar um paralelo entre esta "softwarização da cultura" de Manovich e o conceito da "tecnologia branda" de Paulo Laurentiz (1991). Segundo Manovich, além da cultura estar se tornando software, as próprias funções do hardware se interiorizam (ou virtualizam), levando a uma existência e a interações cada vez mais "moles" ("soft"), e talvez assim mais humanas. Laurentiz sugere um percurso similar, ao falar do "abrandamento" da relação homem-máquina, que permitiria ao Humano se sobressair à técnica.
} 
Nas palavras de Manovich:

Do início dos anos 90 até a metade da década de 2000, o software cultural substituiu a maior parte das demais tecnologias de mídia que emergiram nos séculos 19 e 20. A maior parte da cultura atual é criada e acessada através do software cultural - e ainda assim, surpreendentemente, poucas pessoas conhecem a sua história.9 (MANOVICH, 2008)

\section{Novas Mídias (Procedurais)}

O termo "novas mídias" ("new media") é utilizado para denominar todo conteúdo midiático que se baseia fundamentalmente no numérico e nas características que lhe são próprias.

Assim, enquanto o cinema e a música estão associados a mídias tradicionais, jogos, comunicação online e outros conteúdos procedimentais tratam-se de novas mídias.

Esta nova categoria se diferencia das mídias tradicionais principalmente pelo seu caráter dinâmico (em oposição a estático ou fixo) e interativo. Como vimos, estas características tomam como base um aspecto fundamental do meio numérico, que é o da proceduralidade.

A atual situação midiática é marcada pela riqueza e complexidade das tecnologias, linguagens e práticas, e pelo constante fluxo de novos usos e combinações entre estes elementos. Assim, o termo "novas mídias" continua atual, apesar deste processo já não ser tão novo assim.

\footnotetext{
9 Este documento digital não possui numeração de páginas. Texto original: "Between early 1990 s and middle of the 2000s, cultural software has replaced most other media technologies that emerged in the 19th and 20th century. Most of today's culture is created and accessed via cultural software - and yet, surprisingly, few people know about its history."
} 
É importante não confundir as novas mídias com mídias tradicionais que simplesmente tomam o meio digital como suporte. Como explicado anteriormente, para se configurar como "nova mídia", um conteúdo precisa empregar, ou tomar como base, características que são próprias do numérico, e fazê-lo de uma maneira fundamental.

Um filme, por exemplo, pode ser reproduzido em um computador. Porém, o uso que esta mídia faz dos recursos numéricos não é fundamental no que concerne à natureza do conteúdo em si.

\section{Analítica Cultural / Produção Cultural}

Uma das áreas na qual Manovich e seu grupo de pesquisa tem concentrado as suas explorações práticas é na Analítica Cultural (Cultural Analytics). Trata-se de uma aplicação específica na área da visualização de dados, voltada aos objetos culturais.

No trecho a seguir o autor exemplifica:

Digamos que eu esteja interessado em pensar sobre estratégias cinemáticas em vídeos criados pelos usuários no YouTube. Não há como examinar manualmente todos os bilhões de vídeos ali. (...) [M]eu laboratório já está trabalhando [na busca de] uma maneira de rastrear e analisar cultura em uma nova escala que envolve centenas de milhões de produtores e bilhões de objetos de mídia10. (MANOVICH, 2008)

Note que é possível considerar este tipo de análise de produtos culturais como um processo inverso ao da sua produção. Isto é,

\footnotetext{
10 Texto original: "Let's say I am interested in thinking about cinematic strategies in user-generated videos on YouTube. There is no way I can manually look through all the billions of videos there. (...) [M]y lab is already working on how we can track and analyze culture at a new scale that involve hundreds of millions of producers and billions of media objects."
} 
considerando a existência de um algoritmo capaz de analisar determinadas características em um conjunto de obras, por exemplo, a princípio seria possível aplicá-lo de maneira inversa para produzir uma obra semelhante.

Tomemos como exemplo a análise dos padrões gráficos das capas da revista Time realizada por Manovich e seu grupo, tomando como base 4535 edições da revista, de 1923 a 2009. A princípio, seria possível relacionar de alguma forma as informações obtidas através desta análise ao que seria o seu processo inverso - que, neste caso, poderia ser a direção de arte aplicada ao projeto gráfico da revista, por exemplo.

Com isso, eventualmente seria possível criar um algoritmo capaz de produzir uma típica capa da revista Time a partir de imagens e textos originais (ou arbitrários), inclusive com a possibilidade de escolher o visual de uma década específica (ou talvez até mesmo misturar estilos de diferentes décadas), por exemplo.

Embora não seja nosso objetivo neste artigo aprofundar em questões específicas como esta, este exemplo põe em evidência a natureza das novas mídias, e como elas diferem de maneira significativa e fundamental em relação às mídias tradicionais.

\section{Estudos do Software: panorama atual}

A iniciativa dos Estudos do Software promove periodicamente palestras, exposições e workshops, além das publicações a ela associadas.

No Brasil, a décima edição do FILE (Festival Internacional de Linguagem Eletrônica, em São Paulo), em 2009, contou com Cicero Silva e Lev Manovich, que participaram com textos no catálogo e outras atividades. 
Vale mencionar que muitas vezes as palestras promovidas pela iniciativa no Brasil contam com a participação de outros membros do grupo que residem no exterior, por meio de videoconferência.

No site oficial dos Estudos do Software11 é possível obter informações atualizadas a respeito das suas atividades, como novos lançamentos, experimentos, palestras e demais apresentações, tanto no Brasil quanto no exterior.

\section{O processo criativo e as poéticas do digital}

A abordagem proposta pela disciplina dos Estudos do Software serve de referência e contexto para a minha pesquisa de mestrado, atualmente em andamento, intitulada Máquina Criadora: o Processo Criativo e Poéticas do Digital. Nela, proponho uma investigação das correspondências e relações entre $o$ Processo Criativo e a lógica procedimental do numérico nas Poéticas do Digital. Nosso objetivo é contribuir com as discussões a respeito do uso do computador como meio expressivo, bem como da criatividade de uma maneira mais ampla.

Na pesquisa, em primeiro lugar propomos uma investigação do Processo Criativo em Meios Digitais, principalmente a partir de Julio Plaza e Monica Tavares (PLAZA \& TAVARES, 1998), e examinamos a influência das ferramentas e demais participantes nestas práticas artísticas. Em seguida, tratamos do conceito de Graus de Contribuição (MACHADO, 1993) e sugerimos uma classificação de Camadas de Influência, a partir da qual definimos uma Metodologia de Análise (FERREIRA \& PRADO, 2009).

\section{Máquina Criadora}

${ }^{11}$ lab.softwarestudies.com 
As Poéticas do Digital diferem das manifestações artísticas digitais de uma maneira geral por serem próprias e exclusivas a este meio, tomando como base o aspecto procedimental do numérico.

Propomos o termo "Máquina Criadora" para denominar a situação na qual determinado dispositivo, programa ou algoritmo criado por um artista (ou sob sua orientação) tem a capacidade de gerar uma obra de arte ou executar determinada operação poética. Note que isso não implica necessariamente na existência de algum tipo de inteligência (ou criatividade) artificial - a máquina não é necessariamente criativa, e sim criadora.

Este uso do computador não é novo, e remonta aos primórdios da arte computacional, na década de 60 (escolhemos o termo "Máquina Criadora" unicamente por motivos didáticos, como maneira de delimitar esta prática que adotamos como objeto de estudo).

A discussão a respeito do aspecto procedimental do numérico também não é novidade. Janet Murray trata disso em seu livro Hamlet no Holodeck, cuja primeira edição é de 1997. Porém, como nos mostra Michael Mateas, o uso verdadeiramente expressivo do meio digital ainda é pouco explorado entre os artistas (MATEAS, 2005).

\section{Contextualização e Abordagem}

O processo de "softwarização" da cultura, da qual trata Manovich, está intimamente relacionado ao tema da nossa pesquisa. À medida que a cultura se torna software, ela se torna programável, e com isso determinados aspectos da atividade humana se vêem intrinsecamente associados à lógica do numérico.

Este processo não aconteceu de uma hora para a outra. Inicialmente, mudanças físicas no hardware eram necessárias para modificar ou 
acrescentar novas funcionalidades à máquina - estes dois aspectos se viam fortemente atrelados. Gradualmente, funções de hardware passaram a ser representadas logicamente, via software, levando a um grau de customização, flexibilidade e controle cada vez maior, acessível diretamente ao programador e, posteriormente, aos próprios usuários.

Adotamos a abordagem dos Estudos do Software como contexto para o nosso estudo de forma a examinar e refletir a respeito do processo criativo em meios digitais com ênfase no uso expressivo da tecnologia e nas implicações e significados que identificamos no relacionamento entre o artista e a máquina.

Além dos diálogos com autores, publicações e outras referências diretamente associadas aos Estudos do Software, fundamentais à nossa abordagem, o enfoque particular desta disciplina permite uma leitura única de produções das mais diferentes áreas, inclusive lançando novos olhares em textos já conhecidos.

Também esperamos poder contribuir para o enriquecimento desta área de pesquisa que tomamos como contexto, e que as reflexões e idéias apresentadas possam levar a novas associações, eventualmente servindo de ponto de partida para futuras explorações.

Nosso objeto de estudo é o processo criativo no qual processos mentais do artista se vêem de alguma forma representados na lógica numérica. A partir deste intrigante diálogo entre a subjetividade humana e a objetividade digital, também pretendemos nos aproximar da reflexão mais ampla a respeito da expressão criativa e artística.

Nosso tema é a relação homem-máquina; por isto, importa destacar não o valor calculável, quantitativo, da informação, mas sim o seu valor qualitativo, propriamente incomensurável, que se encontra no âmago da relação entre o homem e a máquina. E, para apreendê-lo, nada melhor do 
que começarmos pelo ponto de encontro entre a arte e a tecnologia. (SANTOS, 1994:46)

\section{Referências}

ECO, Umberto. Obra Aberta: Forma e Indeterminação nas Poéticas Contemporâneas. Coleção Debates. Editora Perspectiva, São Paulo, 9a edição, 2003.

FERREIRA, Daniel e Prado, Gilberto dos Santos. O processo criativo em meios digitais: uma metodologia de análise. In Anais do XVIII Encontro Nacional da ANPAP, Salvador, 2009.

FULLER, Matthew (editor). Software Studies: a Lexicon. Software Studies. The MIT Press, Massachusetts, 2008.

FULLER, Matthew et al. Series - software studies, Setembro 2009. URL: mitpress. mit.edu/catalog/browse/browse.asp?btype $=6 \&$ serid $=179$. Texto de apresentação da série Software Studies da MIT Press. Acessado em Outubro de 2010.

LAURENTIZ, Paulo. A Holarquia do Pensamento Artístico. Editora da UNICAMP, Campinas, 1991.

MACHADO, Arlindo. Máquina e Imaginário: o Desafio das Poéticas Tecnológicas. Editora USP, São Paulo, 1993.

MANOVICH, Lev. The Language of New Media. The MIT Press, Massachusetts, 2001.

MANOVICH, Lev. Software takes command, 2008. URL: lab.softwarestudies.com/2008/11/softbook.html. Publicado online. Documento digital sem numeração de páginas. Acessado em Outubro de 2010. 
MATEAS, Michael e Stern, Andrew. Procedural authorship: A case-study of the interactive drama façade. In Digital Arts and Culture: Digital Experience: Design, Aesthetics, Practice (DAC 2005), Copenhagen, Denmark, 2005.

MURRAY, Janet $\mathrm{H}$. Hamlet no Holodeck: o Futuro da Narrativa no Ciberespaço. Editora UNESP, 2003. Itaú Cultural.

PLAZA, Julio e Tavares, Monica. Processos Criativos Com os Meios Eletrônicos: Poéticas Digitais. Editora Hucitec, São Paulo, 1998.

SANTOS, Laymert. O homem e a máquina. Revista Imagens, (3):46-49, Dezembro 1994. Editora da UNICAMP. 